\title{
Impact of Polyethylene Eye Covers Dressing On Preventing Corneal Complications On Critically Ill Patients At Assiut University Hospital
}

\author{
Asmaa Atiaa Tolba, Mona Aly Mohammed, Mervat Anwar AbdEl Aziz \& Tark Ahmed Mohamed. \\ Demonstrator of Critical Care Nursing, Faculty of nursing, Assiut University Egypt. \\ Lecturer of Critical Care Nursing, Faculty of nursing, Assiut University Egypt. \\ Lecturer of Critical Care Nursing, Faculty of nursing, Assiut University Egypt. \\ Assistant Professor of Ophthalmology, Faculty of medicine, Assiut University Egypt.
}

\begin{abstract}
Prevention of corneal complications for intensive care unit patients is effective way to avoid corneal damage. Polyethylene covering is transparent dressing creates moist chamber providing a barrier against tear-film evaporation and providing physical barrier to organisms Aim: this study was carried out to investigate the impact of polyethylene eye covers dressing on preventing corneal complications. Design: A quasi-experimental design. Setting: Trauma Intensive Care Unit at Assiut University Hospital. Subjects: A convenience sample of 120 eyes of 60 adults patients divided into study and control groups (30 patient for each). Tools: tool I Patient assessment sheet. Tool II: Richmond Agitation Sedation Scale. Tool III: Eye assessment tool. Tool IV: Eye care technique, eye lid closure and eye cover by using polyethylene eye cover. Methods: Researcher assessed each patient's eye and ability to maintain eyelid closure. Then, eye care technique was applied, eye lid closure and eye cover by using polyethylene cover to each patient's eye for preventing corneal complications. Results: Finding of present study revealed that there was significant statistical difference between both study and control groups in $7^{\text {th }}$ day in relation to corneal ulcer in right eye and left eye $(\mathrm{P}=0.001 \& 0.000)$ respectively .Conclusion: eye lid closure and eye cover by using polyethylene eye cover could be significantly effective in preventing corneal complications.
\end{abstract}

\section{Key Words :The Polyethylene Eye Cover, Corneal Complications \& Critically Ill Patient}

\section{Introduction}

Critically ill patients often have impaired systemic and ocular protective mechanisms as a result of impaired mental status, mechanical ventilation therapy or neuromuscular blockage, metabolic derangements, and multiple organ dysfunction. Such patients are at increased risk of ocular surface disorders, which, if not resolved, can result in serious visual impairment (Kam, et al., 2011\& Kocaçal, et al., 2011\& Grixti. et al., 2012). Medical and nursing staffs in intensive care unit (ICU) concentrate the majority of their efforts on life-threatening problems (Edwards et al., 2007)

Incomplete lid closure (lagophthalmos) is the major predisposing factor for corneal complications. It removes the physical and chemical protections of the eye lids, tears, and conjunctiva. Critically ill patients frequently have poor eye lid closure and a reduced ability to use the protective blink reflex due to altered level of conscious as a result of an underlying medical condition such as a head injury, and the effects of sedation and muscle relaxants used to enable other aspects of care (Andrea et al., 2008 \& Elliott, 2010 \& Kam et al., 2011). Defective eyelid closure arising from orbicularis weakness can lead to corneal abrasion, corneal ulceration, scarring and visual loss if treatment is delayed. In addition, critically ill patients often have fluid imbalance and increased vascular permeability, which can cause conjunctival edema that hinders eye lid closure (Jamie et al., 2008\& Lane, 2012).

Conjunctival edema or chemosis, also termed the "ventilator eye," is a common, potentially serious complication in critically ill patients, arising from the adverse physiological effects of mechanical ventilation, The use of positive end-expiratory pressure (PEEP) of $5 \mathrm{cmH}_{2} \mathrm{O}$ and above, tight endotracheal tube taping, fluid overload, increased capillary permeability, and compromised venous return the ocular region due to positive pressure ventilation. This leads to sequestration of fluid in the periocular tissues and chemosis (McHugh et al., 2008 \& Kam et al., 2011 \& Grixti et al., 2012 \&Alansari et al., 2013 \& van et al., 2013).

Corneal abrasions are disruptions of the integrity of the corneal epithelium that generally heal rapidly, usually within 24 to 72 hours. Corneal abrasions in the ICU are often caused as a complication of incomplete eye lid closure, which allows the cornea to dry and therefore jeopardizes the integrity of the eye's epithelium. (Ramirez et al., 2008). A corneal ulcer is an open sore that forms on the cornea, usually caused by infections. Factors that increase risk of developing ulcers include eyelids that do not close completely, dry eye, recent infection or injury 
to eye, weakened immune system, such can be caused by human immunodeficiency virus (HIV), trauma, ocular surface disease, and ocular surgery. Other identified risk factors include age, gender and smoking (Web, 2011).

Eye assessment provides a baseline assessment, monitor response to clinical treatment, identify changes or concerns to the condition of the eyes. Each eye should be assessed independently. Eye assessment in ICU should include eye opening, reaction, risk factors for incomplete eyelid closure, eyelid position, blinking reflex, pupil size, ocular surface dryness, eyelid cleanliness, signs of corneal complications (Ramírez et al., 2008 \& Yi, 2009 \& Demirel et al., 2014).

Eye care is an important aspect of care to maintain the integrity of the ocular surface (Dawson, 2005). Eye care is carried out to clean the eye of discharge and crusts; prior to eye drop installation; to soothe eye irritation; to prevent corneal damage/abrasion in the unconscious or sedated patient because secretions collect along the lid margins and inner canthus when blink reflex is absent or when the eye does not close completely (Andrea et al., 2008 \& Potter, 2011\& Werli-Alvarenga et al., 2011). When suctioning a patient, the nurse should always cover the eye of the side that she is suctioning with gauze, to prevent ocular contamination from the oral flora (Ramírez et al., 2008). The role of critical care nurse providing fundamental eye care to critically ill patients is essential and particularly important in the first 2-7 days, when the peak incidence of corneal eye injury occurs (Andrea et al., 2008).

Polyethylene eye cover is an eye protector that prevents tears from evaporating away from the eye surface. It is also a natural protector that is applied to the front of the eye and preventing possible translocation of infections from sources such as the respiratory tract. There is a transparent film containing $100 \%$ polyethylene in the middle of the cover and double-sided and adhesive drape forms the edges of the cover. Polyethylene can provide various benefits for the ocular surface. For instance, it is waterproof, and has a hypoallergenic adhesive that gently adheres to the skin and provides a natural protection against nosocomial infection agents and the corneal epithelium remains intact when it is used. Polyethylene is also very easy and convenient to use (Shan et al., 2010\& Kocaçal et al., 2011\& Prakash, 2013).

\section{Operational definition}

The polyethylene covering is transparent film dressing creates a moist chamber providing a barrier against tear-film evaporation and exposure to air currents. It may also keep the eye clean and closed by providing a physical barrier to organisms and organisms and preventing possible corneal complications.

\begin{abstract}
Aim of study
The aim of this study is to evaluate the impact of polyethylene eye covers dressing on preventing corneal complications among critically ill patients at Assiut University Hospital.
\end{abstract}

\section{Patients \& methods}

\section{Research design}

Quasi-experimental research design was used to conduct this study. This design was used to explain relationships, clarify certain events happened or both.

\section{Setting}

This study was carried out in Trauma Intensive Care Unit (10 bed) at Assiut University Hospital.

\section{Sampling}

A convenience sample of 120 eyes of 60 male and female patients. Sample was assigned to two equal groups each group consist of 30 patients. Both groups were received routine hospital eye care. Then, the study group subjects eye cover with polyethylene dressing more than this.

\section{Hypothesis}

To fulfill the aim of this study the following research hypothesis is formulated:-

- Corneal complications among critically ill patients who apply polyethylene eye covers dressing in addition to the routine hospital care is significantly less than that among those who received routine eye care only.

\section{Inclusion criteria}

The study included patients had the following criteria

- Recent admission to trauma intensive care unit.

- Age 18 to 60 years.

- Unconscious patient who did not have spontaneous eye opening, opened their eyes briefly in response to stimuli, such as during suctioning, and the frequency of eye opening was limited.

Tools

Four tools were used by the researcher for data collection which are;

Tool one: - Patient assessment sheet:

- This tool was developed by the researcher after reviewing of literatures (Smeltzer et al 2004\& Craven et al 2007\&Pierce, 2007\& Green, 2011), used to assess patient condition, and divided into three parts:

Part I: Socio demographic data and clinical data assessment sheet

Socio demographic data included patient's code, age, sex. Clinical data as diagnosis, type of trauma, past history of diseases, and length of stay in Trauma ICU 
(Craven et al., 2007). In additional to, assessment of Glasgow coma scale (GCS), to evaluate effect of altered in level of conscious in protective mechanism of eye (Green, 2011).

Part II: Assessment of fluid balance \& central venous pressure

This part was used to assess intake\& output and central venous pressure (CVP) to evaluate effect in protective mechanism of eye (Smeltzer et al 2004).

\section{Part III: Assessment of oxygenation:}

This part included type of oxygenation (mechanical ventilation (MV) \& $\mathrm{T}$ - piece connected to venti). Parameters of mechanical ventilation included positive end expiratory pressure (PEEP), fraction of inspired oxygen (FiO2) (Pierce, 2007). In addition to, oxygen concentration $(\mathrm{O} 2)$ for patient ventilated with $\mathrm{T}$ - piece connected to venti.

Tool Two: Richmond Agitation Sedation Scale (RASS)

This tool was adopted from (Sessler et al., 2002) and used to assess patient's anxiety and agitation. One for an alert, calm state and further levels for quality of sedation, which consist of a ten point, with four levels of anxiety or agitation from +1 to +4 [combative]), one level to denote a calm and alert state (0), and 5 levels of sedation from -1 to -5 culminating in unarousable $(-5)$.

In additional to, Sedation and muscles relaxants data was used to assess effect of sedation and muscles relaxant on corneal exposure on critically ill patients. It included drug names and action (Koroloff et al., 2004).

Tool three: Eye assessment tool

This tool was developed by the researcher after reviewing of literatures (Lightman, 2005 \& Elliss, 2006 \& Ramírez et al., 2008 \& Shan et al., 2010; Shojania et al., 2010), and divided to three parts:-

\section{Part I: Eye assessment sheet}

This part used to assess patient's eyelids, conjunctiva, cornea, and pupil from the first day of admission for seven consequent days, and three times per day. Note the following:

- Eyelids: Lesions, crusting, redness, swelling / bruising, and lacerations

- Conjunctiva

- Normal: translucent, flat, sclera visible beneath

- Abnormal: chemosis (edema), discharge, subconjunctival hemorrhage, lacerations, and lesions

- Assess Corneal for

- Normal: - clear, bright, smooth surface.

- Abnormal: Corneal abrasion and corneal ulcer by using fluorescein eye stain test (Elliss, 2006).

\section{Part II: - Eye Grading Guide}

This part was adopted from (Lightman, 2005) and used to accurately assess the degree of eye exposure from the first day of admission for seven consequent days, and three times per day. It consists of three grades:-

- Grade 1 -Lids completely closed.

- Grade 2 - Any conjunctival exposure as shown by any white of the eye being visible, but no corneal exposure.

- Grade 3 - Any corneal exposure, even a very tiny amount.

Tool four: Eye care technique and eye lid closure and eye cover by using polyethylene eye cover

This tool was developed by the researcher after reviewing of literatures (Dawson, 2005; Feroz et al., 2013). This tool was used to maintain the integrity of the ocular surface, and to prevent corneal complications in critically ill patients.

Method

- The study was conducted throughout four main phases, which were preparatory , assessment, implementation and evaluation phases:-

- Preparatory phase

- Permission to conduct the study obtained from the responsible hospital authorities in anesthesiology department, trauma intensive care unit.

- Protection of human rights (ethical considerations): Informed consent was obtained from the responsible person for the unconscious patients and the confidentiality and anonymity of the subjects assured through coding the data.

- The tools (I, III, IV) used in this study were developed by the researcher based on reviewing the relevant literature.

- Content validity: The developed tools (I, III, IV) were tested for content validity by a jury of (7) specialists in the field of critical care nursing and ophthalmology from Assiut University, and necessary modifications were done.

- The Reliability was done on The developed tools (I, III, IV) by Cronbach`s Alpha and reliability level was 0.831to assess the consistency and stability of the tools.

- Pilot study: a pilot study carried out in order to assess the feasibility and applicability of the tools and the necessary modifications were done. The pilot study was done on 6 patients who were excluded from the study.

Data collection

- Data were collected in twelve months approximately.

- The data were collected from the first day of admission after stabilization of the patient's condition and for seven consequent days, every day and every shift then the data were recorded in the developed tools. 
- Assessment phase for control and study group

- During this phase the researcher assessed patient from the first day of admission and record patient socio demographic and clinical data before any data collection by taking this information from his/her sheet using tool 1 ( part 1). Also, assessing Glasgow coma scale one time daily by using tool 1(part 1).

- The researcher assessed homodynamic state of patient one time daily by using tool 1(part 2), also assessed type of oxygenation, mechanical ventilation data daily by using tool 1 (part 3 ). Sedation and muscles relaxant drugs data or anxiety and agitation level assessed daily to determine quality of sedation by using RASS (tool 2).

- The researcher assessed patient's ability to maintain eyelid closure to determine degree of eye exposure from the first day of admission for seven consequent days, and every shift by using tool 3 (part 2).

- The researcher assessed each patient's eye independently (examine lids, conjunctiva, and pupil) from the first day of admission for seven consequent days, and every shift by using tool 3 (part1).

- The fluorescein eye stain test used by researcher from the first day of admission for seven consequent days, and three times per day. This is a test that uses orange dye (fluorescein) and pen torch to detect damage to the cornea as the following:

- Moist the end of the fluorescein strip with Sterile $0.9 \%$ sodium chloride or artificial tears.

- Put a piece of blotting paper containing the dye (fluorescein strip) on the surface of patient's eye, then was waiting seconds until spread of the dye and coated the tear film covering the surface of the cornea.

- Then shone pen torch at patient's eye. Any problems on the surface of the cornea stained by the dye and appear green under the pen torch.

- Then determined damage of the cornea (corneal abrasion or corneal ulcer) (Knoop et al., 2009).

- Implementation phase for study group

- Eye care technique, eye lid closure \& eye cover by using polyethylene cover applied by researcher to each patient's eye independently as the following:

- Clean eyes with sterile normal saline and gauze prior to the application of any eye care product.

- Then, wipe the external eye with normal saline soaked gauze from inner canthus to outer (Elliss, 2006 \& Kocaçal et al., 2011).

- Then, apply polyethylene eye cover to each patient's eye independently to maintain closed eye lid of critically ill patients to prevent corneal complications (Urden et al., 2014).

- Then, change polyethylene eye cover every shift and or as needed if they became unclean or torn (Shan et al., 2010).

- Evaluation phase

This phase was done to evaluate effect of applying eye care technique, eye lid closure and eye cover by using polyethylene cover on preventing corneal complication by using fluorescein eye stain test.

\section{Limitation of the study}

The result of this study could not be generalized due to the small sample size, not representative of all population, not geographically distributed because of the convenience sample.

\section{Statistical analysis}

- Data were computerized and analyzed by computer programme SPSS (ver.16). Data were presented using descriptive statistics in the form of frequencies and percentages or means \pm standard deviations for qualitative data. Quantitative data were compared using Independent samples t- test for comparisons among two groups. Qualitative variables were compared using chi-square test to determine significance.

- The critical value of the tests "P" was considered statistically significant when $\mathrm{P}$ less than 0.05 


\section{Results}

Table (1): Differences of socio-demographic and clinical data of study \& control groups.

\begin{tabular}{|c|c|c|c|c|c|}
\hline & \multicolumn{2}{|c|}{ Study $(n=30)$} & \multicolumn{2}{|c|}{ Control $(n=30)$} & \multirow{2}{*}{ P-value } \\
\hline & No. & $\%$ & No. & $\%$ & \\
\hline \multicolumn{5}{|l|}{ Age } & \multirow{5}{*}{0.987} \\
\hline $18-<30$ years & 18 & 60.0 & 17 & 56.7 & \\
\hline $30-<40$ years & 4 & 13.3 & 4 & 13.3 & \\
\hline $40-<50$ years & 4 & 13.3 & 4 & 13.3 & \\
\hline $50-60$ years & 4 & 13.3 & 5 & 16.7 & \\
\hline Mean \pm SD & \multicolumn{2}{|c|}{$33.18 \pm 11.90$} & \multicolumn{2}{|c|}{$31.47 \pm 12.54$} & 0.598 \\
\hline \multicolumn{5}{|l|}{ Sex } & \multirow{4}{*}{0.421} \\
\hline Male & 28 & 93.3 & 25 & 83.3 & \\
\hline Female & 2 & 6.7 & 5 & 16.7 & \\
\hline \multicolumn{5}{|l|}{ Diagnosis } & \\
\hline Acute respiratory failure & 6 & 20.0 & 4 & 13.3 & 0.488 \\
\hline Head injury & 23 & 76.7 & 22 & 73.3 & 0.766 \\
\hline Spinal cord injury & 0 & 0.0 & 1 & 3.3 & 0.313 \\
\hline Brain tumor & 0 & 0.0 & 1 & 3.3 & 0.313 \\
\hline Acute renal failure & 0 & 0.0 & 1 & 3.3 & 0.313 \\
\hline GIT bleeding & 0 & 0.0 & 1 & 3.3 & 0.313 \\
\hline Post arrest & 1 & 3.3 & 2 & 6.7 & 0.554 \\
\hline \multicolumn{5}{|l|}{ Type of trauma } & \multirow{4}{*}{0.247} \\
\hline Blunt & 27 & 90.0 & 22 & 73.3 & \\
\hline Penetrating & 2 & 6.7 & 5 & 16.7 & \\
\hline Non-traumatic & 1 & 3.3 & 3 & 10.0 & \\
\hline \multicolumn{5}{|l|}{ Length of stay } & \multirow{4}{*}{0.286} \\
\hline $7-<10$ days & 8 & 26.7 & 5 & 16.7 & \\
\hline $10-<15$ days & 12 & 40.0 & 9 & 30.0 & \\
\hline$\geq 15$ days & 10 & 33.3 & 16 & 53.3 & \\
\hline Mean \pm SD & \multicolumn{2}{|c|}{$18.59 \pm 4.74$} & \multicolumn{2}{|c|}{$19.36 \pm 5.12$} & 0.557 \\
\hline
\end{tabular}

Ns: There is no significant difference $p$. value $>0.05 *$ Significant difference at $p$. value $<0.05$

- Independent samples t-test for comparing two groups - Chi-square test for qualitative variables

Table (2): Differences of the study and control groups in relation to fluid balance and central venous pressure mean values.

\begin{tabular}{|l|c|c|c|}
\hline \multirow{2}{*}{} & Study $(\mathbf{n}=\mathbf{3 0})$ & Control(n= 30) & \multirow{2}{*}{ P-value } \\
\cline { 2 - 3 } & Mean \pm SD & Mean \pm SD & \\
\hline Fluid balance & $2157.67 \pm 1529.63$ & $1706.00 \pm 933.79$ & 0.225 \\
\hline 1st day & $1671.33 \pm 1079.91$ & $1503.67 \pm 1492.39$ & 0.307 \\
\hline 4th day & $1911.67 \pm 1210.71$ & $1528.00 \pm 1144.88$ & 0.145 \\
\hline 7th day & $11.20 \pm 4.60$ & $8.16 \pm 4.73$ & $0.017^{*}$ \\
\hline CVP: & $10.64 \pm 3.27$ & $8.52 \pm 4.54$ & $0.046^{*}$ \\
\hline $1^{\text {st }}$ day & $11.75 \pm 3.89$ & $8.58 \pm 3.15$ & $0.009^{*}$ \\
\hline $4^{\text {th }}$ day & \multicolumn{3}{|l}{}
\end{tabular}

Ns: There is no significant difference $p$. value $>0.05 *$ Significant difference at $p$. value $<0.05$

- Independent samples t-test

CVP: central venous pressure 
Table (3): Differences of study and control groups in relation to assessment of oxygenation.

\begin{tabular}{|c|c|c|c|c|c|}
\hline \multirow{2}{*}{ Mode } & \multicolumn{2}{|c|}{ Study $(n=30)$} & \multicolumn{2}{|c|}{ Control $(n=30)$} & \multirow{2}{*}{ P-value } \\
\hline & No. & $\%$ & No. & $\%$ & \\
\hline \multicolumn{6}{|l|}{$1^{\text {st }}$ day } \\
\hline MV & 30 & 100.0 & 28 & 93.3 & \\
\hline PEEP: Mean \pm SD & \multicolumn{2}{|c|}{$6.00 \pm 1.82$} & \multicolumn{2}{|c|}{$5.68 \pm 1.39$} & 0.432 \\
\hline $\mathrm{FiO}_{2} \quad$ Mean \pm SD & \multicolumn{2}{|c|}{$41.33 \pm 5.56$} & \multicolumn{2}{|c|}{$44.29 \pm 6.34$} & 0.060 \\
\hline T-piece with venti & 0 & 0.0 & 2 & 6.7 & \\
\hline$-\mathrm{O}_{2} \quad$ Mean $\pm \mathrm{SD}$ & \multicolumn{2}{|c|}{--} & \multicolumn{2}{|c|}{$60.00 \pm 0.00$} & -- \\
\hline \multicolumn{6}{|l|}{$4^{\text {th }}$ day: } \\
\hline MV & 28 & 93.3 & 26 & 86.7 & \\
\hline PEEP: Mean \pm SD & \multicolumn{2}{|c|}{$5.96 \pm 2.17$} & \multicolumn{2}{|c|}{$5.69 \pm 1.38$} & 0.955 \\
\hline $\mathrm{FiO}_{2}$ Mean \pm SD & \multicolumn{2}{|c|}{$47.68 \pm 12.51$} & \multicolumn{2}{|c|}{$42.31 \pm 5.14$} & $0.047 *$ \\
\hline T-piece with venti & 2 & 6.7 & 4 & 13.3 & \\
\hline $\mathrm{O}_{2} \quad$ Mean $\pm \mathrm{SD}$ & \multicolumn{2}{|c|}{$60.00 \pm 0.00$} & \multicolumn{2}{|c|}{$60.00 \pm 0.00$} & \\
\hline \multicolumn{6}{|l|}{$7^{\text {th }}$ day: } \\
\hline MV & 22 & 73.3 & 22 & 73.3 & \\
\hline PEEP: Mean \pm SD & \multicolumn{2}{|c|}{$5.86 \pm 1.78$} & \multicolumn{2}{|c|}{$5.82 \pm 1.50$} & 0.817 \\
\hline $\mathrm{FiO}_{2} \quad$ Mean \pm SD & \multicolumn{2}{|c|}{$44.45 \pm 6.34$} & \multicolumn{2}{|c|}{$44.45 \pm 7.39$} & 0.986 \\
\hline T-piece with venti & 8 & 26.7 & 8 & 26.7 & \\
\hline $\mathrm{O}_{2} \quad$ Mean $\pm \mathrm{SD}$ & \multicolumn{2}{|c|}{$40.00 \pm 0.00$} & \multicolumn{2}{|c|}{$40.00 \pm 0.00$} & \\
\hline
\end{tabular}

Ns; There is no significant difference $p$. value>0.05* Significant difference at $p$ value $<0.05$ - Independent samples t-test

- MV: mechanical ventilation

- T-piece with venti: venti connected to T- piece

- PEEP: Positive End Expiratory Pressure - FiO2: fraction of inspired oxygen.

- O2: Oxygen

Table (4): Differences of study and control groups according to Glasgow coma scale (GCS).

\begin{tabular}{|l|c|c|c|c|}
\hline \multirow{2}{*}{ GCS $^{*}$} & \multicolumn{2}{c|}{ Study } & \multicolumn{2}{c|}{ Control } \\
\cline { 2 - 5 } & No. & $\%$ & No. & \% \\
\hline \hline $\mathbf{1}^{\text {st }}$ day: & $(\mathrm{n}=14)$ & ----- & $(\mathrm{n}=18)$ & 100.0 \\
\hline GCS: $3-8$ & 14 & 100.0 & 18 & \\
\hline $\mathbf{4}^{\text {th }}$ day: & $(\mathrm{n}=22)$ & & $(\mathrm{n}=23)$ & 100.0 \\
\hline GCS: $3-8$ & 22 & 100.0 & 23 & --- \\
\hline $\mathbf{7}^{\text {th }}$ day: & $(\mathrm{n}=24)$ & ---- & $(\mathrm{n}=25)$ & 100.0 \\
\hline GCS: $3-8$ & 24 & 100.0 & 25 & 1 \\
\hline
\end{tabular}

Ns: There is no significant difference p. value $>0.05 \quad *$ Significant difference at $p$. value $<0.05$

- Chi-square test

- GCS*: Glasgow coma scale was not applicable for sedated patients.

Table (5): Differences of study group and control group in relation to sedation and muscles relaxants medications that directly effecting on eye.

\begin{tabular}{|c|c|c|c|c|c|}
\hline \multirow{2}{*}{ Sedation and muscles relaxants } & \multicolumn{2}{|c|}{ Study $(n=30)$} & \multicolumn{2}{|c|}{ Control $(n=30)$} & \multirow{2}{*}{ P-value } \\
\hline & No. & $\%$ & No. & $\%$ & \\
\hline \multicolumn{6}{|l|}{$1^{\text {st }}$ day } \\
\hline Sedation & 16 & 53.3 & 12 & 40.0 & 0.301 \\
\hline Muscles relaxant & 0 & 0.0 & 0 & 0.0 & -- \\
\hline Both sedation and muscles relaxant & 0 & 0.0 & 2 & 6.7 & 0.472 \\
\hline
\end{tabular}




\begin{tabular}{|c|c|c|c|c|c|}
\hline \multirow{2}{*}{ Sedation and muscles relaxants } & \multicolumn{2}{|c|}{ Study $(n=30)$} & \multicolumn{2}{|c|}{ Control $(n=30)$} & \multirow{2}{*}{ P-value } \\
\hline & No. & $\%$ & No. & $\%$ & \\
\hline \multicolumn{6}{|l|}{$4^{\text {th }}$ day } \\
\hline Sedation & 8 & 26.7 & 7 & 23.3 & 0.766 \\
\hline Muscles relaxant & 0 & 0.0 & 2 & 6.7 & 0.472 \\
\hline Both sedation and muscles relaxant & 0 & 0.0 & 1 & 3.3 & 0.313 \\
\hline \multicolumn{6}{|l|}{$7^{\text {th }}$ day } \\
\hline Sedation & 6 & 20.0 & 5 & 16.7 & 0.739 \\
\hline Muscles relaxant & 0 & 0.0 & 0 & 0.0 & -- \\
\hline Both sedation and muscles relaxant & 0 & 0.0 & 2 & 6.7 & 0.472 \\
\hline
\end{tabular}

Ns: There is no significant difference p. value $>0.05 \quad *$ Significant difference at $p$. value $<0.05$

- Chi-square test

Table (6): Differences of study group and control group in relation to Richmond Agitation Sedation Scale (RASS).

\begin{tabular}{|c|c|c|c|c|c|}
\hline \multirow{2}{*}{ RASS } & \multicolumn{2}{|c|}{ Study } & \multicolumn{2}{|c|}{ Control } & \multirow{2}{*}{ P-value } \\
\hline & No. & $\%$ & No. & $\%$ & \\
\hline $1^{\text {st }}$ day & $(n=16)$ & --- & $(n=12)$ & --- & --- \\
\hline Unarousable & 9 & 56.2 & 2 & 16.7 & 0.054 \\
\hline Deep sedation & 5 & 31.2 & 6 & 50.0 & 0.441 \\
\hline Moderate sedation & 0 & 0.0 & 4 & 33.3 & $0.024 *$ \\
\hline Light sedation & 2 & 12.0 & 0 & 0.0 & 0.492 \\
\hline Drowsy & 0 & 0.0 & 0 & 0.0 & -- \\
\hline $4^{\text {th }}$ day & $(n=8)$ & --- & $(n=7)$ & --- & --- \\
\hline Unarousable & 2 & 25.0 & 2 & 28.6 & 0.876 \\
\hline Deep sedation & 6 & 75.0 & 2 & 28.6 & 0.132 \\
\hline Moderate sedation & 0 & 0.0 & 3 & 42.9 & 0.077 \\
\hline Light sedation & 0 & 0.0 & 0 & 0.0 & -- \\
\hline Drowsy & 0 & 0.0 & 0 & 0.0 & -- \\
\hline $7^{\text {th }}$ day & $(n=6)$ & --- & $(n=5)$ & --- & --- \\
\hline Unarousable & 2 & 33.3 & 0 & 0.0 & 0.455 \\
\hline Deep sedation & 2 & 33.3 & 2 & 40.0 & 0.819 \\
\hline Moderate sedation & 2 & 33.3 & 2 & 40.0 & 0.819 \\
\hline Light sedation & 0 & 0.0 & 1 & 20.0 & 0.455 \\
\hline Drowsy & 0 & 0.0 & 0 & 0.0 & -- \\
\hline
\end{tabular}

Ns: There is no significant difference p. value $>0.05 *$ Significant difference at p. value $<0.05 \quad$ - Chi-square test 
Table (7): Differences of study and control groups according to eye grading guide.

\begin{tabular}{|c|c|c|c|c|c|c|c|c|c|c|}
\hline \multirow{3}{*}{ EGG } & \multicolumn{4}{|c|}{ Right } & \multirow{3}{*}{ P-value } & \multicolumn{4}{|c|}{ Left } & \multirow{3}{*}{ P-value } \\
\hline & \multicolumn{2}{|c|}{ Study $(n=30)$} & \multicolumn{2}{|c|}{ Control $(n=30)$} & & \multicolumn{2}{|c|}{$\operatorname{Study}(n=30)$} & \multicolumn{2}{|c|}{$\operatorname{Control}(n=30)$} & \\
\hline & No. & $\%$ & No. & $\%$ & & No. & $\%$ & No. & $\%$ & \\
\hline \multicolumn{11}{|l|}{$1^{\text {st }}$ day: } \\
\hline Grade I & 21 & 70.0 & 22 & 73.3 & 0.774 & 22 & 73.3 & 22 & 73.3 & -- \\
\hline Grade II & 0 & 0.0 & 2 & 6.7 & 0.472 & 2 & 6.7 & 1 & 3.3 & 0.554 \\
\hline Grade III & 9 & 30.0 & 6 & 20.0 & 0.371 & 6 & 20.0 & 7 & 23.3 & 0.754 \\
\hline \multicolumn{11}{|l|}{$2^{\text {nd }}$ day } \\
\hline Grade I & 20 & 66.7 & 20 & 66.7 & -- & 20 & 66.7 & 20 & 66.7 & -- \\
\hline Grade II & 3 & 10.0 & 3 & 10.0 & -- & 4 & 13.3 & 2 & 6.7 & 0.667 \\
\hline Grade III & 7 & 23.3 & 7 & 23.3 & -- & 6 & 20.0 & 8 & 26.7 & 0.542 \\
\hline \multicolumn{11}{|l|}{$3^{\text {rd }}$ day } \\
\hline Grade I & 20 & 66.7 & 18 & 60.0 & 0.592 & 20 & 66.7 & 19 & 63.3 & 0.787 \\
\hline Grade II & 3 & 10.0 & 3 & 10.0 & -- & 4 & 13.3 & 1 & 3.3 & 0.350 \\
\hline Grade III & 7 & 23.3 & 9 & 30.0 & 0.559 & 6 & 20.0 & 10 & 33.3 & 0.243 \\
\hline \multicolumn{11}{|l|}{$4^{\text {th }}$ day } \\
\hline Grade I & 18 & 60.0 & 18 & 60.0 & -- & 20 & 66.7 & 18 & 60.0 & 0.592 \\
\hline Grade II & 5 & 16.7 & 5 & 16.7 & -- & 4 & 13.3 & 3 & 10.0 & 0.688 \\
\hline Grade III & 7 & 23.3 & 7 & 23.3 & -- & 6 & 20.0 & 9 & 30.0 & 0.371 \\
\hline \multicolumn{11}{|l|}{$5^{\text {th }}$ day } \\
\hline Grade I & 20 & 66.7 & 18 & 60.0 & 0.592 & 22 & 73.3 & 18 & 60.0 & 0.273 \\
\hline Grade II & 3 & 10.0 & 4 & 13.3 & 0.688 & 3 & 10.0 & 4 & 13.3 & 0.688 \\
\hline Grade III & 7 & 23.3 & 8 & 26.7 & 0.766 & 5 & 16.7 & 8 & 26.7 & 0.347 \\
\hline \multicolumn{11}{|l|}{$6^{\text {th }}$ day } \\
\hline Grade I & 24 & 80.0 & 17 & 56.7 & 0.052 & 26 & 86.7 & 19 & 63.3 & $0.037 *$ \\
\hline Grade II & 0 & 0.0 & 4 & 13.3 & $0.037^{*}$ & 0 & 0.0 & 3 & 10.0 & 0.236 \\
\hline Grade III & 6 & 20.0 & 9 & 30.0 & 0.371 & 4 & 13.3 & 8 & 26.7 & 0.197 \\
\hline \multicolumn{11}{|l|}{$7^{\text {th }}$ day } \\
\hline Grade I & 24 & 80.0 & 15 & 50.0 & $0.015^{*}$ & 26 & 86.7 & 16 & 53.3 & $0.005^{*}$ \\
\hline Grade II & 0 & 0.0 & 3 & 10.0 & 0.236 & 0 & 0.0 & 3 & 10.0 & 0.236 \\
\hline Grade III & 6 & 20.0 & 12 & 40.0 & 0.091 & 4 & 13.3 & 11 & 36.7 & $0.037 *$ \\
\hline
\end{tabular}

Ns: There is no significant difference p. value $>0.05 \quad *$ Significant difference at $p$. value $<0.05$

- Chi-square test

Table (8): Differences of study and control groups according to eyelids assessment.

\begin{tabular}{|c|c|c|c|c|c|c|c|c|c|c|}
\hline \multirow{3}{*}{ Eyelids } & \multicolumn{4}{|c|}{ Right } & \multirow{3}{*}{ P-value } & \multicolumn{4}{|c|}{ Left } & \multirow{3}{*}{ P-value } \\
\hline & \multicolumn{2}{|c|}{ Study $(n=30)$} & \multicolumn{2}{|c|}{ Control $(n=30)$} & & \multicolumn{2}{|c|}{ Study $(n=30)$} & \multicolumn{2}{|c|}{ Control $(n=30)$} & \\
\hline & No. & $\%$ & No. & $\%$ & & No. & $\%$ & No. & $\%$ & \\
\hline \multicolumn{11}{|l|}{$1^{\text {st }}$ day } \\
\hline Normal & 22 & 73.3 & 22 & 73.3 & -- & 22 & 73.3 & 22 & 73.3 & -- \\
\hline Redness & 8 & 26.7 & 3 & 10.0 & 0.095 & 5 & 16.7 & 1 & 3.3 & 0.197 \\
\hline $\begin{array}{l}\text { Swelling/ } \\
\text { bruising }\end{array}$ & 0 & 0.0 & 5 & 16.7 & 0.062 & 3 & 10.0 & 7 & 23.3 & 0.166 \\
\hline \multicolumn{11}{|l|}{$2^{\text {nd }}$ day } \\
\hline Normal & 22 & 73.3 & 23 & 76.7 & 0.766 & 22 & 73.3 & 22 & 73.3 & -- \\
\hline Redness & 8 & 26.7 & 3 & 10.0 & 0.095 & 5 & 16.7 & 1 & 3.3 & 0.197 \\
\hline $\begin{array}{l}\text { Swelling/ } \\
\text { bruising }\end{array}$ & 0 & 0.0 & 4 & 13.3 & 0.121 & 3 & 10.0 & 7 & 23.3 & 0.166 \\
\hline
\end{tabular}




\begin{tabular}{|c|c|c|c|c|c|c|c|c|c|c|}
\hline \multirow{3}{*}{ Eyelids } & \multicolumn{4}{|c|}{ Right } & \multirow{3}{*}{ P-value } & \multicolumn{4}{|c|}{ Left } & \multirow{3}{*}{ P-value } \\
\hline & \multicolumn{2}{|c|}{ Study $(n=30)$} & \multicolumn{2}{|c|}{ Control(n= 30) } & & \multicolumn{2}{|c|}{ Study $(n=30)$} & \multicolumn{2}{|c|}{ Control $(n=30)$} & \\
\hline & No. & $\%$ & No. & $\%$ & & No. & $\%$ & No. & $\%$ & \\
\hline \multicolumn{11}{|l|}{$3^{\text {rd }}$ day } \\
\hline Normal & 22 & 73.3 & 24 & 80.0 & 0.542 & 22 & 73.3 & 22 & 73.3 & -- \\
\hline Redness & 8 & 26.7 & 2 & 6.7 & $0.038^{*}$ & 5 & 16.7 & 1 & 3.3 & 0.197 \\
\hline $\begin{array}{l}\text { Swelling/ } \\
\text { bruising }\end{array}$ & 0 & 0.0 & 4 & 13.3 & 0.121 & 3 & 10.0 & 7 & 23.3 & 0.166 \\
\hline \multicolumn{11}{|l|}{$4^{\text {th }}$ day } \\
\hline Normal & 24 & 80.0 & 25 & 83.3 & 0.739 & 24 & 80.0 & 22 & 73.3 & 0.542 \\
\hline Redness & 6 & 20.0 & 1 & 3.3 & 0.108 & 5 & 16.7 & 0 & 0.0 & 0.062 \\
\hline $\begin{array}{l}\text { Swelling/ } \\
\text { bruising }\end{array}$ & 0 & 0.0 & 4 & 13.3 & 0.121 & 1 & 3.3 & 8 & 26.7 & $0.030^{*}$ \\
\hline \multicolumn{11}{|l|}{$5^{\text {th }}$ day } \\
\hline Normal & 24 & 80.0 & 26 & 86.7 & 0.488 & 24 & 80.0 & 23 & 76.7 & 0.754 \\
\hline Redness & 6 & 20.0 & 1 & 3.3 & 0.108 & 5 & 16.7 & 0 & 0.0 & 0.062 \\
\hline $\begin{array}{l}\text { Swelling/ } \\
\text { bruising }\end{array}$ & 0 & 0.0 & 3 & 10.0 & 0.236 & 1 & 3.3 & 7 & 23.3 & 0.058 \\
\hline \multicolumn{11}{|l|}{$6^{\text {th }}$ day } \\
\hline Normal & 24 & 80.0 & 26 & 86.7 & 0.488 & 24 & 80.0 & 23 & 76.7 & 0.754 \\
\hline Redness & 6 & 20.0 & 1 & 3.3 & 0.108 & 5 & 16.7 & 0 & 0.0 & 0.062 \\
\hline $\begin{array}{l}\text { Swelling/ } \\
\text { bruising }\end{array}$ & 0 & 0.0 & 3 & 10.0 & 0.236 & 1 & 3.3 & 7 & 23.3 & 0.058 \\
\hline \multicolumn{11}{|l|}{$7^{\text {th }}$ day } \\
\hline Normal & 25 & 83.3 & 27 & 90.0 & 0.704 & 24 & 80.0 & 22 & 73.3 & 0.542 \\
\hline Redness & 5 & 16.7 & 1 & 3.3 & 0.197 & 5 & 16.7 & 0 & 0.0 & 0.062 \\
\hline $\begin{array}{l}\text { Swelling/ } \\
\text { bruising }\end{array}$ & 0 & 0.0 & 2 & 6.7 & 0.472 & 1 & 3.3 & 8 & 26.7 & $0.030 *$ \\
\hline
\end{tabular}

Ns: There is no significant difference p. value $>0.05 \quad *$ Significant difference at $p$. value $<0.05$

- Chi-square test

Table (9): Differences of study and control groups according to conjunctiva assessment.

\begin{tabular}{|c|c|c|c|c|c|c|c|c|c|c|}
\hline \multirow{3}{*}{ Conjunctiva } & \multicolumn{4}{|c|}{ Right } & \multirow{3}{*}{ P-value } & \multicolumn{4}{|c|}{ Left } & \multirow{3}{*}{ P-value } \\
\hline & \multicolumn{2}{|c|}{$\begin{array}{c}\text { Study } \\
(n=30)\end{array}$} & \multicolumn{2}{|c|}{$\begin{array}{l}\text { Control } \\
(n=30)\end{array}$} & & \multicolumn{2}{|c|}{$\begin{array}{c}\text { Study } \\
(n=30)\end{array}$} & \multicolumn{2}{|c|}{$\begin{array}{l}\text { Control } \\
(n=30)\end{array}$} & \\
\hline & No. & $\%$ & No. & $\%$ & & No. & $\%$ & No. & $\%$ & \\
\hline \multicolumn{11}{|l|}{$1^{\text {st }}$ day } \\
\hline Normal & 17 & 56.7 & 21 & 70.0 & 0.284 & 13 & 43.3 & 16 & 53.3 & 0.438 \\
\hline Chemosis & 12 & 40.0 & 7 & 23.3 & 0.165 & 16 & 53.3 & 10 & 33.3 & 0.118 \\
\hline Discharge & 1 & 3.3 & 0 & 0.0 & 0.313 & 1 & 3.3 & 0 & 0.0 & 0.313 \\
\hline Subconjunctival hemorrhage & 0 & 0.0 & 2 & 6.7 & 0.472 & 0 & 0.0 & 4 & 13.3 & 0.121 \\
\hline \multicolumn{11}{|l|}{$2^{\text {nd }}$ day } \\
\hline Normal & 13 & 43.3 & 17 & 56.7 & 0.302 & 7 & 23.3 & 15 & 50.0 & $0.032 *$ \\
\hline Chemosis & 16 & 53.3 & 10 & 33.3 & 0.118 & 22 & 73.3 & 10 & 33.3 & $0.002 *$ \\
\hline Discharge & 1 & 3.3 & 1 & 3.3 & -- & 1 & 3.3 & 1 & 3.3 & -- \\
\hline Subconjunctival hemorrhage & 0 & 0.0 & 2 & 6.7 & 0.472 & 0 & 0.0 & 4 & 13.3 & 0.121 \\
\hline \multicolumn{11}{|l|}{$3^{\text {rd }}$ day } \\
\hline Normal & 11 & 36.7 & 17 & 56.7 & 0.121 & 5 & 16.7 & 15 & 50.0 & $0.006^{*}$ \\
\hline Chemosis & 18 & 60.0 & 10 & 33.3 & $0.038 *$ & 24 & 80.0 & 10 & 33.3 & $0.000 *$ \\
\hline Discharge & 1 & 3.3 & 1 & 3.3 & -- & 1 & 3.3 & 1 & 3.3 & -- \\
\hline
\end{tabular}




\begin{tabular}{|c|c|c|c|c|c|c|c|c|c|c|}
\hline \multirow{3}{*}{ Conjunctiva } & \multicolumn{4}{|c|}{ Right } & \multirow{3}{*}{ P-value } & \multicolumn{4}{|c|}{ Left } & \multirow{3}{*}{ P-value } \\
\hline & \multicolumn{2}{|c|}{$\begin{array}{c}\text { Study } \\
(\mathbf{n}=\mathbf{3 0})\end{array}$} & \multicolumn{2}{|c|}{$\begin{array}{l}\text { Control } \\
(n=30)\end{array}$} & & \multicolumn{2}{|c|}{$\begin{array}{c}\text { Study } \\
(n=30)\end{array}$} & \multicolumn{2}{|c|}{$\begin{array}{l}\text { Control } \\
(n=30)\end{array}$} & \\
\hline & No. & $\%$ & No. & $\%$ & & No. & $\%$ & No. & $\%$ & \\
\hline Subconjunctival hemorrhage & 0 & 0.0 & 2 & 6.7 & 0.472 & 0 & 0.0 & 4 & 13.3 & 0.121 \\
\hline \multicolumn{11}{|l|}{$4^{\text {th }}$ day } \\
\hline Normal & 9 & 30.0 & 18 & 60.0 & $0.020 *$ & 7 & 23.3 & 16 & 53.3 & $0.017^{*}$ \\
\hline Chemosis & 20 & 66.7 & 9 & 30.0 & $0.004 *$ & 22 & 73.3 & 9 & 30.0 & $0.001 *$ \\
\hline Discharge & 1 & 3.3 & 1 & 3.3 & -- & 1 & 3.3 & 1 & 3.3 & -- \\
\hline Subconjunctival hemorrhage & 0 & 0.0 & 2 & 6.7 & 0.472 & 0 & 0.0 & 4 & 13.3 & 0.121 \\
\hline \multicolumn{11}{|l|}{$5^{\text {th }}$ day } \\
\hline Normal & 9 & 30.0 & 17 & 56.7 & $0.037 *$ & 7 & 23.3 & 15 & 50.0 & $0.032 *$ \\
\hline Chemosis & 20 & 66.7 & 9 & 30.0 & $0.004 *$ & 22 & 73.3 & 9 & 30.0 & $0.001^{*}$ \\
\hline Discharge & 1 & 3.3 & 1 & 3.3 & -- & 1 & 3.3 & 1 & 3.3 & -- \\
\hline Subconjunctival hemorrhage & 0 & 0.0 & 3 & 10.0 & 0.236 & 0 & 0.0 & 5 & 16.7 & 0.062 \\
\hline \multicolumn{11}{|l|}{$6^{\text {th }}$ day } \\
\hline Normal & 7 & 23.3 & 15 & 50.0 & $0.032 *$ & 7 & 23.3 & 13 & 43.3 & 0.100 \\
\hline Chemosis & 23 & 76.7 & 11 & 36.7 & $0.002 *$ & 23 & 76.7 & 11 & 36.7 & $0.002^{*}$ \\
\hline Discharge & 0 & 0.0 & 1 & 3.3 & 0.313 & 0 & 0.0 & 1 & 3.3 & 0.313 \\
\hline Subconjunctival hemorrhage & 0 & 0.0 & 3 & 10.0 & 0.236 & 0 & 0.0 & 5 & 16.7 & 0.062 \\
\hline \multicolumn{11}{|l|}{$7^{\text {th }}$ day: } \\
\hline Normal & 7 & 23.3 & 17 & 56.7 & $0.008 *$ & 7 & 23.3 & 15 & 50.0 & $0.032 *$ \\
\hline Chemosis & 23 & 76.7 & 10 & 33.3 & $0.001 *$ & 23 & 76.7 & 10 & 33.3 & $0.001 *$ \\
\hline Discharge & 0 & 0.0 & 1 & 3.3 & 0.313 & 0 & 0.0 & 1 & 3.3 & 0.313 \\
\hline Subconjunctival hemorrhage & 0 & 0.0 & 2 & 6.7 & 0.472 & 0 & 0.0 & 4 & 13.3 & 0.121 \\
\hline
\end{tabular}

Ns: There is no significant difference p. value $>0.05 *$ Significant difference at $p$. value $<0.05$ - Chi-square test

Hypothesis: Corneal complications among critically ill patients who apply polyethylene eye covers dressing (study group) for them were significantly less than that among those who received routine eye care (control group).

Table (10): Differences of study and control groups according to cornea assessment.

\begin{tabular}{|c|c|c|c|c|c|c|c|c|c|c|}
\hline \multirow{3}{*}{ Cornea } & \multicolumn{4}{|c|}{ Right } & \multirow{3}{*}{ P-value } & \multicolumn{4}{|c|}{ Left } & \multirow{3}{*}{ P-value } \\
\hline & \multicolumn{2}{|c|}{$\operatorname{Study}(n=30)$} & \multicolumn{2}{|c|}{ Control $(n=30)$} & & \multicolumn{2}{|c|}{ Study $(n=30)$} & \multicolumn{2}{|c|}{$\operatorname{Control}(n=30)$} & \\
\hline & No. & $\%$ & No. & $\%$ & & No. & $\%$ & No. & $\%$ & \\
\hline \multicolumn{11}{|l|}{$1^{\text {st }}$ day: } \\
\hline Normal & 26 & 86.7 & 18 & 60.0 & $0.020^{*}$ & 26 & 86.7 & 16 & 53.3 & $0.005^{*}$ \\
\hline Corneal abrasion & 4 & 13.3 & 9 & 30.0 & 0.117 & 4 & 13.3 & 7 & 23.3 & 0.317 \\
\hline Corneal ulcer & 0 & 0.0 & 3 & 10.0 & 0.236 & 0 & 0.0 & 7 & 23.3 & $0.016 *$ \\
\hline \multicolumn{11}{|l|}{$2^{\text {nd }}$ day } \\
\hline Normal & 26 & 86.7 & 9 & 30.0 & $0.000 *$ & 18 & 60.0 & 9 & 30.0 & $0.020 *$ \\
\hline Corneal abrasion & 0 & 0.0 & 15 & 50.0 & $0.000 *$ & 8 & 26.7 & 10 & 33.3 & 0.573 \\
\hline Corneal ulcer & 4 & 13.3 & 6 & 20.0 & 0.488 & 4 & 13.3 & 11 & 36.7 & $0.037 *$ \\
\hline \multicolumn{11}{|l|}{$3^{\text {rd }}$ day: } \\
\hline Normal & 23 & 76.7 & 8 & 26.7 & $0.000 *$ & 21 & 70.0 & 6 & 20.0 & $0.000 *$ \\
\hline Corneal abrasion & 2 & 6.7 & 10 & 33.3 & $0.010^{*}$ & 5 & 16.7 & 8 & 26.7 & 0.347 \\
\hline Corneal ulcer & 5 & 16.7 & 12 & 40.0 & $0.045^{*}$ & 4 & 13.3 & 16 & 53.3 & $0.001 *$ \\
\hline \multicolumn{11}{|l|}{$4^{\text {th }}$ day } \\
\hline Normal & 22 & 73.3 & 8 & 26.7 & $0.000 *$ & 23 & 76.7 & 6 & 20.0 & $0.000^{*}$ \\
\hline Corneal abrasion & 3 & 10.0 & 9 & 30.0 & 0.053 & 3 & 10.0 & 6 & 20.0 & 0.470 \\
\hline Corneal ulcer & 5 & 16.7 & 13 & 43.3 & $0.024^{*}$ & 4 & 13.3 & 18 & 60.0 & $0.000 *$ \\
\hline
\end{tabular}




\begin{tabular}{|c|c|c|c|c|c|c|c|c|c|c|}
\hline \multicolumn{11}{|l|}{$5^{\text {th }}$ day } \\
\hline Normal & 24 & 80.0 & 8 & 26.7 & $0.000 *$ & 26 & 86.7 & 5 & 16.7 & $0.000 *$ \\
\hline Corneal abrasion & 2 & 6.7 & 9 & 30.0 & $0.020 *$ & 1 & 3.3 & 8 & 26.7 & $0.030 *$ \\
\hline Corneal ulcer & 4 & 13.3 & 13 & 43.3 & $0.010^{*}$ & 3 & 10.0 & 17 & 56.7 & $0.000 *$ \\
\hline \multicolumn{11}{|l|}{ 6 $^{\text {th }}$ day } \\
\hline Normal & 21 & 70.0 & 8 & 26.7 & $0.001 *$ & 25 & 83.3 & 5 & 16.7 & $0.000 *$ \\
\hline Corneal abrasion & 6 & 20.0 & 10 & 33.3 & 0.243 & 3 & 10.0 & 9 & 30.0 & 0.053 \\
\hline Corneal ulcer & 3 & 10.0 & 12 & 40.0 & $0.007 *$ & 2 & 6.7 & 16 & 53.3 & $0.000 *$ \\
\hline \multicolumn{11}{|l|}{$7^{\text {th }}$ day } \\
\hline Normal & 20 & 66.7 & 10 & 33.3 & $0.010^{*}$ & 23 & 76.7 & 7 & 23.3 & $0.000 *$ \\
\hline Corneal abrasion & 8 & 26.7 & 7 & 23.3 & 0.766 & 5 & 16.7 & 6 & 20.0 & 0.739 \\
\hline Corneal ulcer & 2 & 6.7 & 13 & 43.3 & $0.001 *$ & 2 & 6.7 & 17 & 56.7 & $0.000 *$ \\
\hline
\end{tabular}

Ns: There is no significant difference p. value $>0.05 *$ Significant difference at $p$. value $<0.05 \quad$ - Chi-square test

Table (1): illustrates personal and clinical characteristics of study and control groups. Regarding to age, it was noticed that the mean age in study and control groups $(33.18 \pm 11.90$ and $31.47 \pm$ 12.54) respectively. Regarding to sex, the majority of patients were male in study and control groups $(93.3 \%$ and $83.3 \%)$ respectively. As regard to diagnosis, the majority of patients were complained from head injury in study and control groups (76.7\% and $73.3 \%$ ) respectively. Regarding to types of trauma, the majority of patients were having blunt trauma in study and control groups $(90.0 \%$ and $73.3 \%$ ) respectively. As regarding to length of stay, near to half of patients stayed in range of $10 \geq 15$ days (40.0\% and $53.3 \%$ ) respectively. There were no statistically significant difference between study and control groups in relation to age, sex, diagnosis, type of trauma, and length of stay ( $\mathrm{P}$ value $>0.05$ ).

Table (2): shows fluid balance and central venous pressure of the study and control groups. As regards to fluid balance. It was noticed that, there was no statistical significant differences between study and control groups ( $\mathrm{P}$ value $>0.05$ ). Regarding to central venous pressure, results revealed that there was statistical significant differences between study and control groups in $1^{\text {st }}, 2^{\text {nd }}, 3^{\text {rd }}, 4^{\text {th }}, 6^{\text {th }}, 7^{\text {th }}$ days $(\mathrm{P}=$ $0.017 \& \mathrm{P}=\quad 0.042 \& \mathrm{P}=\quad 0.036 \& \mathrm{P}=0.046 \& \mathrm{P}=$ $0.008 \& \mathrm{P}=0.009)$ respectively.

Table (3): shows assessment of oxygenation. As regards to, type of oxygenation, results revealed that the majority of patients were connected to mechanical ventilation in both groups in $1^{\text {st }}$ day $(100.0 \%$ and $93.3 \%)$ respectively and in $7^{\text {th }}$ day (73.3\% and $73.3 \%$ ) respectively. Regarding to Positive End Expiratory Pressure (PEEP), it was noticed that there was no statistical significant differences between study and control groups ( $\mathrm{P}$ value > 0.05). As regard to fraction of inspired oxygen (FiO2), results revealed that there was a statistical significant difference between study and control groups in $4^{\text {th }}$ days $(\mathrm{P}=0.047)$. Regarding to
Oxygen $\left(\mathrm{O}_{2}\right)$, it was noticed that there was no statistical significant differences between study and control groups ( $\mathrm{P}$ value $>0.05)$.

Table (4): shows Glasgow coma scale of study and control groups. It can be noted from this table that, in $1^{\text {st }}$ day 14 and 18 patients of the study and control groups respectively were unconscious. In $4^{\text {th }}$ day that, 22 and 23 patients of the study and control groups respectively were unconscious. In $7^{\text {th }}$ day that, 24 and 25 patients of the study and control groups respectively were unconscious.

Table (5): shows sedation and muscles relaxants that directly effecting on eye.

It was found that there was no a statistical significant difference between study and control groups in relation to sedation and muscles relaxants medications ( $\mathrm{P}$ value $>0.05)$.

Table (6): shows Richmond Agitation Sedation Scale (RASS). Regarding moderate sedation, It was noticed that there was a statistical significant difference between study and control groups in $1^{\text {st }}$ day $(\mathrm{P}=0.024)$. Regarding Unarousable, It was noticed that there was a statistical significant difference between study and control groups in $2^{\text {nd }}$ day $(\mathrm{P}=0.014)$.

Table (7): shows eye grading guide of study and control groups. Regarding Grade I in right eye, it was observed that there was a statistical significant difference between study and control groups $7^{\text {th }}$ day $(P=0.015)$. Also regarding Grade $\mathrm{I}$ in left eye, results revealed that there was a statistical significant difference between study and control groups in $6^{\text {th }}, 7^{\text {th }}$ days $(\mathrm{P}=0.037 \& \mathrm{P}=0.005)$ respectively. Regarding Grade II, it was noticed that there was a statistical significant difference between study and control groups in right eye $6^{\text {th }}$ day $(\mathrm{P}=0.037)$. Regarding Grade III, it was noticed that there was a statistical significant difference between study and control groups in left eye $7^{\text {th }}$ day $(\mathrm{P}=0.037)$

Table (8): shows eyelids assessment of study and control groups. Regarding redness, it was found that 
there was a statistical significant difference between study and control groups in right eye in $3^{\text {rd }}$ day $(\mathrm{P}=$ 0.038). Regarding Swelling/ bruising, it was noticed that there was a statistical significant difference between study and control groups in left eye in $4^{\text {th }}, 7^{\text {th }}$ days $(\mathrm{P}=0.030 \& \mathrm{P}=0.030)$ respectively.

Table (9): shows conjunctiva assessment of study and control groups. Regarding chemosis in right eye, it was observed that there was a statistical significant difference between study and control groups in $3^{\text {rd }}$, $4^{\text {th }}, 5^{\text {th }}, 6^{\text {th }}, 7^{\text {th }}$ days $(\mathrm{P}=0.038 \& \mathrm{P}=0.004 \& \mathrm{P}=$ $0.004 \& \mathrm{P}=0.002 \& \mathrm{P}=0.001)$ respectively. Also, regarding chemosis in left eye, results revealed that there was a statistical significant difference between study and control groups in $2^{\text {nd }}, 3^{\text {rd }}, 4^{\text {th }}, 5^{\text {th }}, 6^{\text {th }}, 7^{\text {th }}$ days $(\mathrm{P}=0.002 \& \mathrm{P}=0.000 \& \mathrm{P}=0.001 \& \mathrm{P}=0.001 \& \mathrm{P}=$ $0.002 \& \mathrm{P}=0.001)$ respectively.

Table (10): shows cornea assessment of study and control groups. Results revealed that more than half of study group patients were have normal cornea in both eyes from $1^{\text {st }}$ to $7^{\text {th }}$ days, and there was a statistical significant difference between study and control groups in $1^{\text {st }}, 2^{\text {nd }}, 3^{\text {rd }}, 4^{\text {th }}, 5^{\text {th }}, 6^{\text {th }}, 7^{\text {th }}$ days $(\mathrm{P}$ right eye $=0.020 \& \mathrm{P}=0.000 \& \mathrm{P}=0.000 \& \mathrm{P}=$ $0.000 \& \mathrm{P}=0.000 \& \mathrm{P}=0.001 \& \mathrm{P}=0.010)$ respectively $\&$ $(\mathrm{P} \quad$ left $\quad$ eye $=\quad 0.005 \&$ $\mathrm{P}=0.020 \& \mathrm{P}=0.000 \& \mathrm{P}=0.000 \& \mathrm{P}=0.000 \& \mathrm{P}=0.000 \& \mathrm{P}$ $=0.000)$ respectively. As regard to corneal abrasion in right eye, it was observed that there was a statistical significant difference between study and control groups in $2^{\text {nd }}, 3^{\text {rd }}, 5^{\text {th }}$ days $(\mathrm{P}=0.000 \& \mathrm{P}=0.010 \& \mathrm{P}=$ 0.020) respectively. Also, regarding corneal abrasion in left eye, it was noticed that there was a statistical significant difference between study and control groups in $5^{\text {th }}$ day $(\mathrm{P}=0.030)$. Regarding corneal ulcer in right eye, results revealed that there was a statistical significant difference between study and control groups in $3^{\text {rd }}, 4^{\text {th }}, 5^{\text {th }}, 6^{\text {th }}, 7^{\text {th }}$ days $(\mathrm{P}=0.045 \& \mathrm{P}=0.024 \& \mathrm{P}=0.010 \& \mathrm{P}=0.007 \& \mathrm{P}=0.001)$ respectively. Also, regarding corneal ulcer in left eye, it was noticed that there was a statistical significant difference between study and control groups in $1^{\text {st }}$, $2^{\text {nd }}, 3^{\text {rd }}, 4^{\text {th }}, 5^{\text {th }}, 6^{\text {th }}, 7^{\text {th }}$ days $(\mathrm{P}=0.016 \& \mathrm{P}=0.037 \& \mathrm{P}=$ $0.001 \& \mathrm{P}=0.000 \& \mathrm{P}=0.000 \& \mathrm{P}=0.000 \& \mathrm{P}=0.000)$ respectively.

\section{Discussion}

Critically ill patients are susceptible to a wide range of corneal complications due to environment, treatment therapy, and clinical prioritization with emphasis on the life rather than sight threatening conditions. (Grixti et al., 2012 \& Alansari et al., 2013). Healthy epithelium of the cornea is a barrier against most infectious agents; dryness-related epithelial breakdown in Intensive Care units (ICU) patients can lead to numerous corneal complications. Protection of the cornea is very important to avoid these complications (Jamie et al., 2008, Ezra et al., 2009\&Kocaçal et al., 2011).

A variety of eye care regimens are available for intensive care patients, but nothing is conclusive so far. The basic principle for preventing corneal complication is meticulous and protocolized care. The aim and objective of intensive nursing care is to provide general and specific critical care plan to each patients (Feroz et al., 2013).

Two eye care methods that are commonly used in ICU to protect eyes are instilling saline or methylcellulose lubricating drops and taping the eyelids in the shut position. Evidence suggests that an alternative technique is more effective in preventing corneal complications. This alternative technique includes cleaning eye with saline and a polyethylene eye cover is taped over the eyes, extending beyond the orbits and eyebrows. Polyethylene eye cover creates a moisture chamber around the cornea and assists in keeping the eyes moist and in the closed position. This technique also prevents damage to the eyes that result from tape or gauze being placed directly on the delicate skin of the eyelids (Urden et al., 2014). This study aimed to evaluate impact of eye care nursing guidelines on preventing corneal complications for critically ill patients.

In our current study, most of patients were male and the mean age in study and control groups (33.18 \pm 11.90 and $31.47 \pm 12.54)$ respectively with no statistically difference between study and control groups. This in according with (Alansari et al., 2013), who found that there were no significant differences in sex and age distribution between patients who developed corneal complications compared to those who did not after applying eye care for them.

In the current study, we documented that the majority of patients diagnosed with head injury and connected to mechanical ventilation which increase risk of development of corneal complications due to altered level of conscious, loss ability to close eye lid completely and a reduced ability to use the protective blink reflex. Similary (Demirel et al., 2014), found that some clinical conditions such as, head or facial trauma, mechanical ventilation, and sedative or neuromuscular blocker treatments which are usually encountered in intensive care units, were reported to cause loss of the eye protective mechanisms and increase the risk of corneal complications which is still reported as an important problem for ICU patients. Also (Werli-Alvarenga et al., 2011), found that the variables that presented significant association with corneal complications were: 
presence of neurological disease, intubation, and mechanical ventilation.

Sedation and decreased consciousness may compromise the random eye movements, cause loss of the blinking reflex and compromise the lacrimal film (Werli-Alvarenga et al., 2013). In the current study, high percent of patients were unconscious so that those patients did not have spontaneous eye opening or may opened their eyes briefly in response to stimuli, such as during suctioning, incomplete eye lid closure and the frequency of blinking was limited so that increase risk for corneal complications as corneal abrasion and corneal ulcer. This result was in line with (Andrea et al., 2008)who found that altered levels of consciousness impact on the protective mechanisms of the eye that increase risk of eye injury, such as corneal dehydration, abrasion, and ulceration. Also (Fiona et al., 2007), found that mechanically ventilated patients who are unconscious are a high risk group who are dependent on eye care to maintain eye integrity. These patients are susceptible to corneal dehydration, abrasions and ulcer as a result of impairment of basic eye protective measures.

In the current study, we found that patients on sedation and muscle relaxants medications were more susceptible to incomplete eye lid closure that contributing in developing corneal complications. This agrees with (Andrea et al., 2008), who found that critically ill patient were unable to maintain normal eye protective mechanisms such as eyelid closure and an intact blink reflex because of the use of sedation and muscle relaxants more susceptible to corneal complications. Also This in line with (Shan, et al., 2010), found that critically ill patients are often paralyzed and sedated leading to incomplete eyelid closure, a loss of the blink reflex and a lack of random eye movements so these patients are susceptible to corneal complications.

In the current study, high present of patients close eye lid completely and frequency of eye opening was limited that also contributing in developing corneal complications due to decrease in tears which lubricate the ocular surface, and providing oxygen to the cornea. In additional to, limited blinking leading to decrease replenishing and spreading the tear film across the cornea. This agrees with (Alansari et al., 2013) who found that long term eye closure reduces tear secretion, causes hypoxia and hypercapnia, and retards reepithelization. Blinking is, therefore, important for distributing tears and maintaining a healthy ocular surface.

The current study presented that the majority of patients on positive pressure ventilation (PEEP) ranged from 5:7 $\mathrm{cmH}_{2} \mathrm{O}$ and all of studied patients on endotracheal tube ties that may increase central venous pressure and contributing in occurrence of conjunctival chemosis and promoting incomplete eyelid closure which increase risk for corneal complications. This result was in line with (Kam et al., 2013), found that elevated central venous pressure as a result of endotracheal tube ties and altered vascular permeability commonly cause conjunctival edema (chemosis), promoting lagophthalmos (incomplete eyelid closure) can result in corneal damage. Also, this agree with (Wilkins et al., 2009) who stated that the use of PEEP of 5 $\mathrm{cmH}_{2} \mathrm{O}$ and above can initiate body fluid retention. Common PEEP setting in critically ill mechanically ventilated patients causes conjunctival chemosis.

In the current study, there was more than half of patients have fluid overload may potentially lead to the conjunctival edema. Also, mechanically ventilated patients extended periods of time in supine position provoke the accumulation of liquid on their face that may lead to conjunctival chemosis so, eye lid does not close completely that contributing in occurrence of corneal complications. This agree with (Ramirez et al., 2008) who documented that patients in the ICU often experience fluid overload, electrolyte problems, and increased permeability. All of these are situations may potentially lead to the conjunctival chemosis. This agree with (McHugh et al., 2008) who studied screening for ocular surface disease in the intensive care unit, documented that patients in the intensive care unit, especially ventilated patients, are at considerable risk of developing corneal complications. Also, found that fluid imbalance and positive pressure ventilation may lead to chemosis. This in line with (Shan et al, 2010), found that critical illness is frequently associated with capillary leak and fluid retention that causes peripheral edema and conjunctival edema and then may lead to inadequate eyelid closure.

In our study, corneal complications were significantly reduced in study group than control group after using of polyethylene eye cover which created a moist chamber and provided a barrier against tear-film evaporation. This agree with (Dawson, 2005) who documented that the use of polyethylene covers is more effective than lubrication in preventing corneal complications. Also, this agree with (Kocaçal et al., 2011) who studied effectiveness of polyethylene eye cover versus carbomer drops to prevent dry eye syndrome in the critically ill and documented that polyethylene maintain the corneal epithelium remains intact. Polyethylene eye cover provides more effective dryness protection than carbomer lubrication for unconscious ICU patients. Also (Shan et al., 2010) conducted a study to compare the efficacy three forms of eye care (artificial tear, moist chamber and polyethylene covers) for intensive care 
patients and noted that polyethylene covers were more effective in reducing the incidence of corneal complications in intensive care patients.

\section{Conclusion}

Based on the results of this study, it could be concluded that: implementing eye lid closure and eye cover by using polyethylene cover are significantly effective in preventing corneal complications in critically ill patients. Polyethylene covers are more effective, easier and more time-saving in preventing corneal damage in intensive care patients.

\section{Recommendations}

Based on the study findings, the following recommendations are suggested:-

- Emphasize the importance of assessing critically ill patient's eyelids, conjunctiva, cornea, and pupil for early detection of any problem.

- Assessment of critically ill patient ability to maintain eyelid closure by Eye Grading Guide should be performed daily in intensive care units.

- Eye care should standardize as a basic part of care provided to all critically ill patients in intensive care units.

- Applying polyethylene eye cover should be standardized in ICU as a moist chamber method of eye care.

\section{References}

1. Alansari M., Hijazi M., \& Maghrabi K., (2013): Making a Difference in Eye Care of the Critically Ill Patients. Journal of Intensive Care Medicine; 1-7.

2. Andrea P., Marshall R., Elliott R., Rolls K., Schacht S., \& Boyle M., (2008): Eye care in the critically ill: Clinical practice Guideline.

3. Craven R., Hirnle C., (2007): Fundamentals of nursing human health and function. 5th ed. USA: Lippincott William\& Wilkins, Inc.

4. Dawson D., (2005): Development of a new eye care guideline for critically ill patients, Intensive and Critical Care Nursing; 21(2):119-122

5. Demirel S., Cumurcu T., Firat P., Said M, Do ganay S., (2014): Effective management of exposure keratopathy developed in intensive care units: The impact of an evidence based eye care education programme, Intensive and Critical Care Nursing 30, 38 - 44

6. Edwards J., Peterson K., Andrus M., Tolson J, Goulding J, Dudeck M., Mincey R., Pollock D., Horan T., (2007): National healthcare safety network report;35(5): 290-301
7. Elliott R., (2010): Provision of Eye Care for the Critically Ill Adult. Intensive care coordination, Monitoring unit; 4.

8. Elliss W., (2006): Eye care for the mechanically ventilated intensive care patients, critical care clinical practices review committee. Central Coast area health; 1-4.

9. Ezra D., Chan M., Solebo L., Malik A., Crane E, Coombes A., Healy M., (2009): randomized trial comparing ocular lubricants and polyacrylamide hydrogel dressings in the prevention exposure keratopathy in the critically ill. Intensive Care Medicine;33:455-61

10. Feroz M., Faisal M., \& Alzeer A., (2013): Protocolized eye care prevents corneal complications in ventilated patients in a medical intensive care unit. Saudi Journal of Anesthesia; 7(1): 33-36.

11. Fiona M., Margaret K,Sharon M., Bronwyn A., (2007): Nursing care of the mechanically ventilated patient: What does the evidence say? Part two, Intensive and Critical Care Nursing; 23, $71-80$

12. Green M., (2011): Cheerio, Laddie! Bidding Farewell to the Glasgow Coma Scale. Annals of Emergency Medicine; 58 (5): 427-430.

13. Grixti A., Sadri M., Edgar J., Datta A., (2012): Uncommon Ophthalmic surface disorders in patients in intensive care units. Journal of Critical Care; 10(1): 26-42.

14. Jamie B., Rosenberg M., Lewis A., Eisen M., (2008): Eye Care in the Intensive Care Unit: Narrative Review and Meta-analysis. Critical Care Medicine ;36(12):3151-3155 .

15. Kam K., Haldar S., Papamichael E., Pearce E., Hayes M., Joshi V., (2013): Eye care in the critically ill: a national survey and protocol. Journal of the Intensive Care Society; 14(2): 150:154

16. Kam M., Hayes M., Joshi N., (2011): Ocular care and complications in the critically ill. Trends in Anesthesia and Critical Care; 257-262.

17. Kocaçal E., Eşer I., \& Eğrilmez S., (2011): Effectiveness of polyethylene covers versus carbomer drops (Viscotears $\left.{ }^{\circledR}\right)$ to prevent dry eye syndrome in the critically ill. Journal of Clinical Nursing; 20: 1916-1922

18. Koroloff N., Boots R., Lipman J., Thomas P., Rickard C., Coyer F., (2004): A randomized controlled study of the efficacy of hypromellose and Lacri-Lube combination versus polyethylene/cling wrap to prevent corneal epithelial breakdown in the semiconscious intensive care patient. Intensive Care Medicine;30(6):1122-6 
19. Lane C., (2012): Management of ocular surface exposure. British Journal Ophthalmology; 96(4)

20. Lightman S., (2005): eye care protocol for patients in Intensive care unit; 2.

21. McHugh J., Alexander P., Kalhoro A., \& Ionides A., (2008): Screening for ocular surface disease in the intensive care unit, eye; 22 : 1465 1468.

22. Pierce L., (2007): Management of the mechanically ventilated patient. $2^{\text {nd }}$ ed. Philadelphia: Elsevier, Inc.

23. Potter A., Griffin A., Stockert P., Hall A., (2011): Basic nursing. $7^{\text {th }}$ ed .Mosby, Inc., 769790.

24. Prakash S., (2013): Perioperative eye protection under general anesthesia. Journal Anesthesiology Clinical Pharmacology; 29(1): 138-139.

25. Ramirez F., Ibarra S., Varon J., Tang R., (2008): The neglected eye: ophthalmologic issues in the intensive care unit. Critical Care\& Shock; 11:72-82.

26. Sessler C., Gosnell M., Grap M., Brophy G., (2002): The Richmond Agitation-Sedation Scale. Validity and reliability in adult intensive care unit patients. American Journal of respiratory and critical care medicine; 166; 1338-1344.

27. Shan H., Du M., (2010): Prevention of exposure keratopathy in intensive care Unit. International Journal of Ophthalmology; 3(4):346-348

28. Shojania K., Duncan B., McDonald K., Wachter R., Markowiz A., (2010): Making health care safer a critical analysis of patient safety practice. Agency for healthcare research and quality (US). Available at: http//archive.ahrq.gov/clinic/ptsafety/.

29. Sivasankar S., Jasper S., Simon S., Jacob P., John G., Raju R., (2006): Eye care in ICU. Indian Journal Critical Care Medicine; 10 :( 1): 11- 4 .

30. Smeltzer S., Bare B., (2004): Brunner\& Suddarth's Text book of medical- surgical nursing.10thed. USA: Lippincott William\& Wilkins; 68.

31. So H., Lee C., Leung A., Lim J., Chan C., Yan W., (2008): comparing the effectiveness of polyethylene covers ( Gladewarp TM) with lanolin (Duratears) eye ointment to prevent corneal abrasions in critically ill patients: a randomized controlled study. The International Journal of Nursing Studies;54:1565-71

32. Urden L., Stacy K., Lough M., (2014): 7th ed Critical care nursing: diagnosis and management. Philadelphia: Mosby Elsevier; p. 648.

33. Van R., Torn E., Ros F., Haas L., (2013): a red eye on the intensive care unit.The journal of medicine; 71(4).
34. Wilkins R., Stoller J., Kacmarek R., (2009): Egan's fundamentals of respiratory care. 9th ed. China: Mosby Elsevier;1028:42

35. Web M., (2011): Corneal Ulcer. available at: http://www.webmd.com/eye-health/corneal-ulcer

36. Werli-Alvarenga A., Ercole F., Herdman T., Chianca T., (2013): Nursing interventions for adult intensive care patients with risk for corneal injury: a systematic review. International Journal Nursing Knowledge; 24(1):25-9

37. Werli-Alvarenga A., Falci F., Antônio F., Aloísio J., Couto T., ( 2011): corneal injuries: incidence and risk factors in the Intensive Care Unit. Revista Latino-Americana Enfermagem; 19(5):1088-95.

38. Yi Y., (2009): Evidence based eye care protocol for ICU patients with altered level of consciousness. Hong Kong University. Published master thesis. The HKU scholars Hub. Hong Kong University. 